\title{
ANGPTL3 possibly promotes cardiac angiogenesis through improving proangiogenic ability of endothelial progenitor cells after myocardial infarction
}

Fei Luo ${ }^{1}$, Panyun $\mathrm{Wu}^{1}$, Jingfei Chen ${ }^{2}$, Yuan Guo ${ }^{1}$, Jiangang Wang ${ }^{3}$, Xiangping $\mathrm{Li}^{1}$ and Zhenfei Fang ${ }^{1 *}$

\begin{abstract}
Angiopoietin Like protein 3 (ANGPTL3) is at present considered as a central molecular target for therapy designed to reduce atherogenic lipids and atherosclerosis. However, concerns about the safety of inactivation of ANGPTL3 in patients with coronary artery disease (CAD) especially myocardial infarction (MI) have been raised. ANGPTL3 is reported to possess proangiogenic property. Angiogenesis is critical to the recovery of MI. Endothelial progenitor cells (EPCS) have multiple differentiation potential and play an important role in the angiogenesis post-Ml. Promoting the function of EPCs could facilitate the angiogenesis and recovery of MI. Previous studies have shown that ANGPTL3 can promote angiogenesis in corneal of rats and promote angiogenesis of endothelial cells by binding to integrin $a_{v} \beta_{3}$ receptors and promoting phosphorylation of protein kinase B (AKT). Our institution found that activated AKT can up-regulate the expression of microRNA-126 (miR-126), which can promote the proangiogenic ability of EPCS. The integrin $a_{v} \beta_{3}$ receptors and AKT also express in EPCs and are closely related to proangiogenic function. Therefore, we hypothesized that ANGPTL3 could improve function of EPCs by binding to integrin $a_{v} \beta_{3}$ receptors and up-regulating miR-126 expression via activating AKT, thus promoting the formation of new blood vessels, attenuating myocardial ischemia and improving heart function.
\end{abstract}

Keywords: ANGPTL3, Angiogenesis, Endothelial progenitor cells, Myocardial infarction

\section{Background}

Coronary artery disease (CAD) is a leading cause of mortality worldwide, myocardial infarction (MI) is one of the most serious type of this disease. Remarkably good results have been achieved in the treatment of CAD by lipid-lowering strategy. Recent research reported individuals with Angiopoietin Like protein 3 (ANGPTL3) loss-of-function mutations demonstrate a $17 \%$ reduction in circulating triglycerides, a $12 \%$ reduction in low-density lipoprotein cholesterol, and a $34 \%$ reduction in odds of CAD, in contrast, high level of ANGPTL3 is positively associated with MI [1]. Inhibition of ANGPTL3 in dyslipidemic mice results in a

\footnotetext{
* Correspondence: fangzhenfei@csu.edu.cn

'Department of Cardiovascular Medicine, The Second Xiangya Hospital, Central South University, 139 Middle Renmin Road, Changsha 410011, Hunan, China

Full list of author information is available at the end of the article
}

dramatically decrease in atherogenic lipids, atherosclerotic lesion area and necrotic content [2]. In view of emerging results of human genetic analysis and preclinical studies, ANGPTL3 is at present considered as a central molecular target for therapy designed to reduce atherogenic lipids [3]. A variety of strategies have been used to genetically or pharmacologically inactivate ANGPTL3. In recent preclinical and clinical studies, CRISPR-Cas9 technology, antisense oligonucleotides and monoclonal antibody have been used to decrease circulating ANGPTL3 concentrations, resulting in an markable decrease in triglyceride-rich lipoprotein as well as low density lipoprotein cholesterol [4-6]. Therefore, ANGPTL3 seems to be a promising target for CAD treatment.

However, concerns about the safety of inactivation of ANGPTL3 in patients with CAD especially MI have been presented [7, 8]. Angiopoietin-like proteins show 
structural similarity to members of angiopoietin family proteins, which are closely related to angiogenesis. ANGPTL3, as one member of the ANGPTL family, is reported to promote angiogenesis and induce blood vessel formation [9]. Angiogenesis, the formation of new capillaries from pre-existing vessels, in the ischemic area after MI promotes cardiomyocyte survival and is believed to be essential to the recovery of patients with MI $[10,11]$. Bonauer et al. [12] reported that inhibition of microRNA-92a enhances recovery of left ventricular function via accelerating blood vessel growth in a mouse model of acute MI. Miyahara et al. [13] reported transplantation of monolayered mesenchymal stem cells induces angiogenesis and improves cardiac function in rats with MI. These studies suggest a critical important role of angiogenesis for cardiac recovery after MI. However, there is little research about the function of ANGPTL3 in cardiac angiogenesis after MI. Previous study reported ANGPTL3 can promote angiogenesis in corneal of rats [9], suggesting that it may also promote cardiac angiogenesis after MI. If ANGPTL3 plays pivotal role in cardiac angiogenesis after MI, the high level of ANGPTL3 in MI patients may be beneficial for cardiac recovery and anti-ANGPTL3 therapy will be disadvantage $[7,8]$. Therefore, we must be cautious with the use of inhibitors of ANGPTL3 and it is also important to illustrate the relationship between ANGPTL3 and cardiac angiogenesis after MI. In this hypothesis, we provide a possible proangiogenic mechanism of ANGPTL3 post-MI.

\section{Presentation of the hypothesis}

Angiogenesis in response to ischemia after MI is critical important and believed to be initiated by endothelial progenitor cells (EPCs). The angiogenesis ability of EPCs mainly depends on its proliferation, adhesion, migration, and in vitro angiogenesis capacity. Previous study reported ANGPTL3 binds to integrin $\alpha_{v} \beta_{3}$ receptor and induces haptotactic endothelial cell adhesion and migration [9]. The angiogenesis associated receptor integrin $\alpha_{v} \beta_{3}$ is a kind of integrin receptor and expressed in cardiovascular endothelial cells. Integrin $\alpha_{v} \beta_{3}$ also was reported to express in EPCs and contribute to its migration [14]. Therefore, ANGPTL3 may also bind to the integrin $\alpha_{v} \beta_{3}$ in EPCs and facilitate EPCs to adhere and migrate.

Besides, ANGPTL3 could stimulate phosphorylation of $\mathrm{AKT}$ in human microvascular vein endothelial cells which contributes to its proangiogenic ability [9]. Our institution found that activated protein kinase B (AKT) signaling pathway increases the expression of microRNA-126 (miR-126) in EPCs and thereby improving angiogenesis [15]. It was reported transplantation of mesenchymal stem cells overexpressing miR-126 improves angiogenesis and cardiac function in MI mice
$[16,17]$. Consequently, we believe that ANGPTL3 can induce miR-126 expression by stimulating the AKT pathway.

Taken together, we hypothesize ANGPTL3 improves proangiogenic ability of EPCs via binding to integrin $\alpha_{v} \beta_{3}$ and increasing expression of miR-126 with an aim of providing more evidence for exploring the proangiogenic function of ANGPTL3 and insight into novel therapeutic target.

\section{Testing the hypothesis}

We will design some experiments to test this hypothesis. (1) C57BL/6 mice will be randomly treated with placebo or Angptl3 recombinant protein or monoclonal antibody, evinacumab, against Angptl3. After 24 h' treatment, they will be conducted with MI surgery by ligating the left anterior descending coronary artery. After that, mice will continue the previous treatment for several days. At the end of the experiment, we will evaluate whether the recombinant protein can improve the cardiac function, infarct area and capillary density and illuminate the safety of evinacumab treatment in MI mice. Capillary density was determined by immunohistochemical staining with CD31 antibody as previously described [15]. We will also detect expression of integrin $\alpha_{v} \beta_{3}$ receptors, phosphorylated AKT, and miR-126. (2) We will treat bone marrow-derived EPCs obtained from MI mice with or without ANGPTL3 recombinant protein, then detect the migratory ability, proliferative ability and in vitro angiogenesis of EPCs and also measure the expression of integrin $\alpha_{v} \beta_{3}$ receptors, phosphorylated AKT and miR-126.

\section{Abbreviations}

AKT: Protein kinase B; ANGPTL3: Angiopoietin Like protein 3; CAD: Coronary artery disease; EPCs: Endothelial progenitor cells; MI: Myocardial infarction; miR-126: MicroRNA-126

\section{Funding}

This work was supported by the grants from Hunan Provincial Innovation Foundation for Postgraduate (Recipient: Fei Luo), Hunan Provincial Natural Science Foundation of China (No. 2018JJ2587, Recipient: Zhenfei Fang) and the National Natural Science Foundation of China (No. 81570267, Recipient: Zhenfei Fang).

\section{Authors' contributions}

ZF and FL conceived the idea; FL and PW wrote the manuscript; FL, PW, JC, $Y G, J W$ and $X L$ collected and read the literature; $Z F$ read through and corrected the manuscript. All authors read and approved the final manuscript.

Ethics approval and consent to participate Not applicable.

Consent for publication Not applicable.

Competing interests

The authors declare that they have no competing interests. 


\section{Publisher's Note}

Springer Nature remains neutral with regard to jurisdictional claims in published maps and institutional affiliations.

\section{Author details}

'Department of Cardiovascular Medicine, The Second Xiangya Hospital, Central South University, 139 Middle Renmin Road, Changsha 410011, Hunan, China. ${ }^{2}$ Department of Obstetrics and Gynecology, Xiangya Hospital, Central South University, Changsha 410011, Hunan, China. ${ }^{3}$ Department of Cardiovascular Medicine, The Third Xiangya Hospital, Central South University, Changsha 410011, Hunan, China.

Received: 1 April 2018 Accepted: 26 July 2018

Published online: 07 August 2018

\section{References}

1. Stitziel NO, Khera AV, Wang $X$, et al. ANGPTL3 deficiency and protection against coronary artery disease. J Am Coll Cardiol. 2017;69:2054-63.

2. Dewey FE, Gusarova V, Dunbar RL, et al. Genetic and pharmacologic inactivation of ANGPTL3 and cardiovascular disease. N Engl J Med. 2017;377: 211-21.

3. Su X, Peng DQ. New insights into ANGPLT3 in controlling lipoprotein metabolism and risk of cardiovascular diseases. Lipids Health Dis. 2018;17:12.

4. Chadwick AC, Evitt NH, Lv W, et al. Reduced blood lipid levels with in vivo CRISPR-Cas9 base editing of ANGPTL3. Circulation. 2018;137:975-7.

5. Graham MJ, Lee RG, Brandt TA, et al. Cardiovascular and metabolic effects of ANGPTL3 antisense oligonucleotides. N Engl J Med. 2017;377:222-32.

6. Gaudet D, Gipe DA, Pordy R, et al. ANGPTL3 inhibition in homozygous familial hypercholesterolemia. N Engl J Med. 2017;377:296-7.

7. Luo F, Guo Y, Fang Z, et al. Concerns on the genetic or therapeutic antagonism of ANGPTL3. J Am Coll Cardiol. 2017;70:2099.

8. Luo F, Guo Y, Li X, et al. Oligonucleotides targeting ANGPTL3. Int J Cardiol 2018;256:8.

9. Camenisch G, Pisabarro MT, Sherman D, et al. ANGPTL3 stimulates endothelial cell adhesion and migration via integrin alpha vbeta 3 and induces blood vessel formation in vivo. J Biol Chem. 2002;277:17281-90.

10. Cochain C, Channon KM, Silvestre JS. Angiogenesis in the infarcted myocardium. Antioxid Redox Signal. 2013;18:1100-13.

11. Guo Y, Luo F, Liu Q, et al. Regulatory non-coding RNAs in acute myocardial infarction. J Cell Mol Med. 2017;21:1013-23.

12. Bonauer A, Carmona G, Iwasaki M, et al. MicroRNA-92a controls angiogenesis and functional recovery of ischemic tissues in mice. Science. 2009;324:1710-3

13. Miyahara $Y$, Nagaya N, Kataoka M, et al. Monolayered mesenchymal stem cells repair scarred myocardium after myocardial infarction. Nat Med. 2006; 12:459-65.

14. Moon HE, Byun K, Park HW, et al. COMP-Ang1 potentiates EPC treatment of ischemic brain injury by enhancing angiogenesis through activating AKTmTOR pathway and promoting vascular migration through activating Tie2FAK pathway. Exp Neurobiol. 2015:24:55-70

15. Guo Y, Luo F, Zhang $X$, et al. TPPU enhanced exercise-induced epoxyeicosatrienoic acid concentrations to exert cardioprotection in mice after myocardial infarction. J Cell Mol Med. 2018;22:1489-500.

16. Chen JJ, Zhou SH. Mesenchymal stem cells overexpressing MiR-126 enhance ischemic angiogenesis via the AKT/ERK-related pathway. Cardiol J. 2011;18:675-81.

17. Huang $F$, Zhu $X, H u X Q$, et al. Mesenchymal stem cells modified with miR126 release angiogenic factors and activate notch ligand Delta-like-4, enhancing ischemic angiogenesis and cell survival. Int J Mol Med. 2013;31: 484-92.

Ready to submit your research? Choose BMC and benefit from:

- fast, convenient online submission

- thorough peer review by experienced researchers in your field

- rapid publication on acceptance

- support for research data, including large and complex data types

- gold Open Access which fosters wider collaboration and increased citations

- maximum visibility for your research: over $100 \mathrm{M}$ website views per year

At $\mathrm{BMC}$, research is always in progress.

Learn more biomedcentral.com/submissions 